\title{
Association of Electrocardiographic P-Wave Markers and Atrial Fibrillation in Embolic Stroke of Undetermined Source
}

\author{
Tony Y.W. Li ${ }^{a}$ Leonard Leong L. Yeo ${ }^{b, c}$ Jamie Sin Ying Ho ${ }^{d}$ Aloysius S. Leow ${ }^{a}$ \\ Mark Y.Chan ${ }^{\text {b, e }}$ Mayank Dalakoti ${ }^{\mathrm{e}}$ Bernard P.L. Chan ${ }^{\mathrm{c}}$ Hock Luen Teoh $^{\mathrm{c}}$ \\ Swee-Chong Seow ${ }^{b}$ e Pipin Kojodjojo ${ }^{\text {b, e }}$ Vijay K. Sharma ${ }^{b, c}$ \\ Benjamin Y.-Q. Tan ${ }^{b, c}$ Ching-Hui Sia ${ }^{b, d}$
}

anternal Medicine Residency, National University Health System, Singapore, Singapore; bepartment of Medicine, Yong Loo Lin School of Medicine, National University of Singapore, Singapore, Singapore; 'Division of Neurology, Department of Medicine, National University Health System, Singapore, Singapore; dDepartment of Medicine, University of Cambridge, Cambridge, UK; eDepartment of Cardiology, National University Heart Centre Singapore, Singapore, Singapore

\section{Keywords}

Atrial fibrillation · Ischaemic stroke $\cdot$ Cerebrovascular disease/stroke $\cdot$ Embolism · P-wave $\cdot$ Electrocardiogram

\begin{abstract}
Background: Several P-wave indices are thought to represent underlying atrial remodeling and have been associated with ischaemic stroke even in the absence of atrial fibrillation (AF). However, the utility of these P-wave indices in predicting outcomes in patients with embolic stroke of undetermined source (ESUS) has not been studied. The aim of this study is to examine these different P-wave indices towards predicting new-onset $\mathrm{AF}$ and stroke recurrence in a cohort of patients with ESUS, thereby demonstrating the value of these electrocardiographic markers for stroke risk stratification. Methods: Between October 2014 and October 2017, consecutive patients diagnosed with ESUS were followed for new-onset $A F$ and ischaemic stroke recurrence. The various $\mathrm{P}$-wave indices, namely, the P-terminal force in the precordial lead V1 (PTFV1), P-wave duration, P-wave dispersion, in-
\end{abstract}

karger@karger.com www.karger.com/ced

Karger $\stackrel{\text { ! }}{\div}$

GOPEN ACCESS
(C) 2020 The Author(s)

Published by S. Karger AG, Basel

This is an Open Access article licensed under the Creative Commons Attribution-NonCommercial-4.0 International License (CC BY-NC) (http://www.karger.com/Services/OpenAccessLicense), applicable to the online version of the article only. Usage and distribution for commercial purposes requires written permission. teratrial blocks, and P-wave axis, were assessed on the initial electrocardiogram on presentation and studied for their relation to eventual AF detection and recurrent stroke. $\boldsymbol{R e}$ sults: 181 ischaemic stroke patients with ESUS were recruited and followed up for a median duration of 2.1 years. An abnormal PTFV1 was associated with occult AF detection but not with recurrent ischaemic strokes. No significant association was observed between the other P-wave indices with either occult AF or stroke recurrence. Conclusion: PTFV1 is associated with AF detection but not recurrent strokes in ESUS patients and can be a useful electrocardiographic marker for further risk stratification in ESUS patients.

(C) 2020 The Author(s)

Published by S. Karger AG, Basel

\section{Introduction}

Strokes are a major cause of morbidity and mortality worldwide [1]. Determining the underlying stroke aetiology is key for secondary prevention. However, there remain a significant population of patients with embolic 
stroke of undetermined source (ESUS) in whom the specific attributable cause of the stroke remains unclear after an extensive evaluation for common aetiologies [2-4]. In these patients, the optimal secondary prevention strategy remains unknown.

It is hypothesized that a proportion of ESUS are cardioembolic in origin contributed by subclinical or paroxysmal atrial fibrillation (AF) [4]. Studies have shown that AF is often diagnosed in ESUS patients after a duration of extended follow-up $[5,6]$. The rate of AF detection improves further with prolonged heart rhythm monitoring and can reach up to $30 \%$ after 3 years of continuous monitoring [7-10]. AF rarely develops in a healthy atrium and typically occurs in the setting of an abnormal atrial substrate where abnormalities such as fibrosis and dilation can lead to electro-anatomical remodeling and contribute towards the pathogenesis of AF [11]. Against this backdrop, there has been growing research towards the use of oral anticoagulation in patients with ESUS. Evidence is however equivocal, and recent studies such as NAVIGATE ESUS [12] and RE-SPECT ESUS [13] did not observe benefit in the use of direct oral anticoagulation. A pertinent question is whether patients were accurately identified with regards to the presence of underlying atrial cardiopathy and if measurements of atrial cardiopathy were sufficiently validated against the risk of stroke recurrence.

Measurements of left atrial (LA) dimensions as well as composite surrogate measures such as the LA volume index (LAVi) have been shown to demonstrate atrial cardiopathy and predict the development of new-onset AF and strokes [14-16]. Although an echocardiographic assessment is part of the work-up for ESUS, it may not always be available in clinical practice at the point of initial patient contact. However, a 12-lead electrocardiogram (ECG) is a simple, easily accessible, and inexpensive tool that is almost universally available. Several ECG indices that are thought to represent underlying atrial remodeling have been associated with stroke and AF independently [17-21]. These measures include the (a) P-terminal force in the precordial lead V1 (PTFV1); (b) P-wave duration (PWD); (c) P-wave dispersion (PWDis); (d) interatrial blocks (IABs); and (e) P-wave axis. We aimed to evaluate these different $\mathrm{P}$-wave indices in predicting new-onset AF and stroke recurrence in a cohort of patients with ESUS, thereby demonstrating the value of these electrocardiographic markers for stroke risk stratification.

\section{Methodology}

\section{Study Design}

This was a retrospective cohort of consecutive patients with ESUS diagnosis at discharge from a stroke unit at a tertiary care hospital, from October 2014 to October 2017. ESUS was diagnosed according to the consensus criteria, defined by the Cryptogenic Stroke/ESUS International Working Group as the presence of non-lacunar ischaemic stroke, absence of atherosclerosis causing $\geq 50 \%$ luminal stenosis, LVEF $\geq 30 \%$, and no identifiable cardioembolic source of embolism [2]. All patients had neuroimaging and vascular studies, 24 -h inpatient cardiac telemetry or Holter monitoring and transthoracic 2D-echocardiography. As part of standard work-up, transthoracic 2D-echocardiography was performed for each patient during the same inpatient stay. Standard measurements of various echocardiographic and Doppler parameters were made in accordance with American Society of Echocardiography guidelines [22]. The echocardiograms were reviewed by a single reader, who was blinded to the baseline clinical data and outcomes. LA and left ventricular parameters measured include LA volume, LAVi, LA diameter, LA diameter index (LADi), left ventricular end-diastolic volume index, and left ventricular mass index.

Patients were followed up for the development of new-onset $\mathrm{AF}$ and ischaemic stroke recurrence. Demographic data such as age, sex, and comorbidities and laboratory results were collected. All patients were followed up for at least 1 year unless death, AF, or recurrent ischaemic stroke occurred. In addition, all patients were offered prolonged cardiac monitoring with an implantable loop recorder. If they declined, detection of AF was done clinically at follow-up appointments. This study was approved by the local institutional review board.

\section{ECG Analysis}

Each of the patients underwent a standard 12-lead surface ECG, which was recorded at a speed of $25 \mathrm{~mm} / \mathrm{s}$ and a voltage of $10 \mathrm{~mm} / \mathrm{mV}$. The ECGs were analysed in a digital format for the morphology and quantitatively for the intervals and all measurements obtained at 4 times digital zoom. All assessments were performed by a single observer blinded to the patient group.

The key ECG parameters assessed were defined as follows and some examples are shown in Figure 1. PTFV1 was defined as the amplitude of the downward deflection of the terminal portion of the P-wave in lead V1 multiplied by its duration [17]. As described in previous studies, PTFV1 was only measured if the P-wave morphology in lead V1 had a negative or biphasic component (shown in Fig. 1a, b respectively) and is excluded when there is a positive $\mathrm{P}$-wave. The $\mathrm{P}$-wave durations were measured in both lead V1 $\left(\mathrm{PWD}_{\mathrm{V} 1}\right)$ and II $\left(\mathrm{PWD}_{\mathrm{II}}\right)$. It was defined as the time from the earliest onset of $\mathrm{P}$-wave activity in the lead to the last $\mathrm{P}$-wave activity in this lead (shown in Fig. 1c). PWDis was calculated by subtracting the minimum $\mathrm{P}$-wave duration from the maximum $\mathrm{P}$-wave duration (shown in Fig. 1d) [18]. An interatrial conduction block was identified based on a $\mathrm{P}$-wave duration $\geq 120 \mathrm{~ms}$. P-wave morphology as previously defined with a biphasic morphology in leads II, III, and aVF or biphasic morphology in leads III and aVF in association with a bifid P-wave in lead II [19]. P-wave axis was determined by measuring the positive or negative $\mathrm{P}$-wave deflections on all $6 \mathrm{limb}$ leads and then calculating the net direction of electric activity using the hex-axial reference system. An abnormal P-wave axis was defined as any value outside $0-75^{\circ}[20]$. 

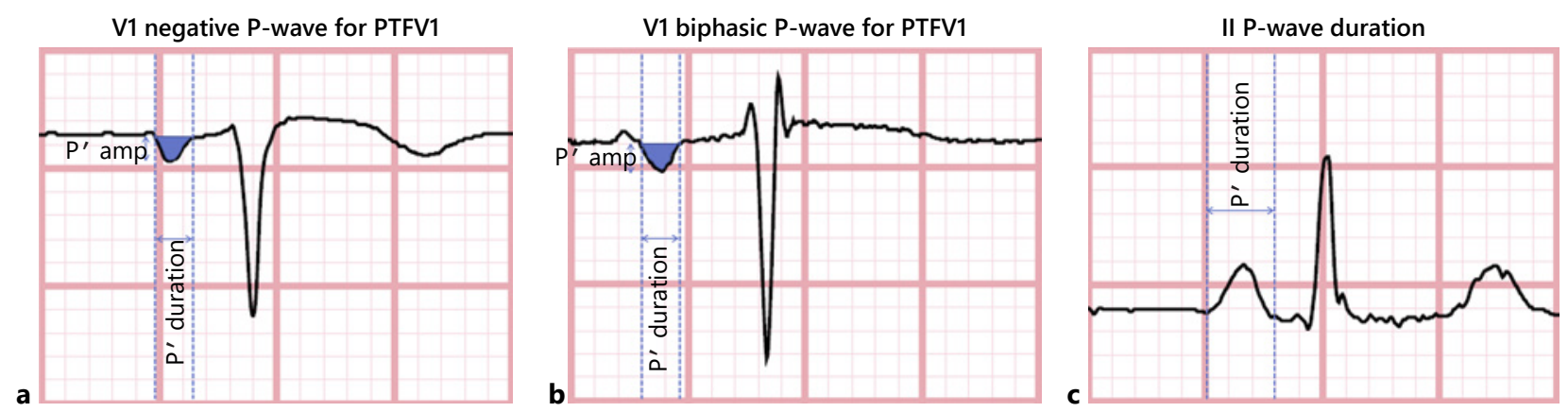

* PTFV1 = P wave Amplitude $\times \mathrm{P}$ wave duration for negative and biphasic $\mathrm{P}$-waves in $\mathrm{V} 1$ but ECGs with postive $\mathrm{P}$-waves in $\mathrm{V} 1$ is excluded

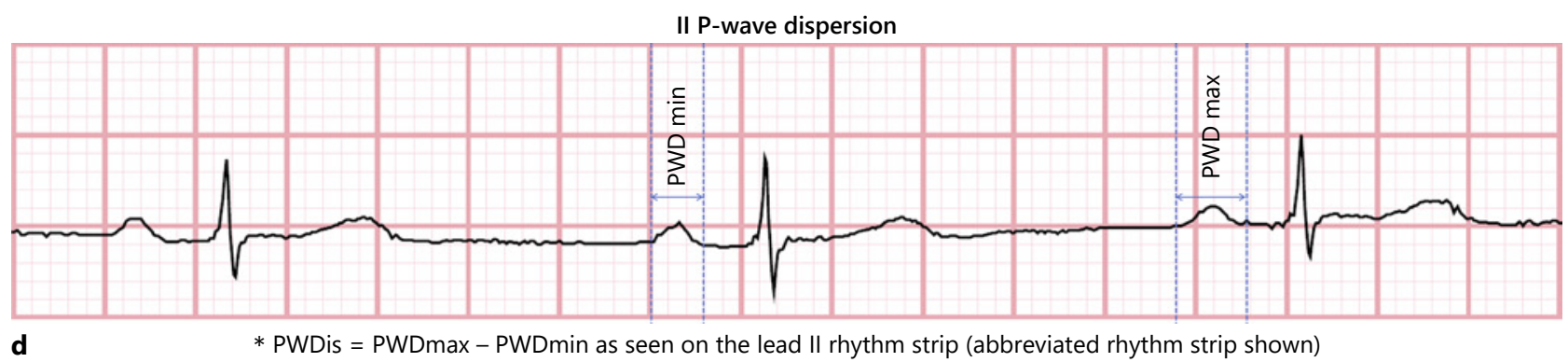

Fig. 1. Measurement criteria for P-wave indices. a Measurement of P-terminal force in the precordial lead V1 (PTFV1) with a negative $\mathrm{P}$-wave in V1. b Measurement of PTFV1 with a biphasic P-wave in V1. c Measurement of the P-wave duration in lead II. d Mea- surement of $\mathrm{P}$-wave dispersion (PWDis) is made by subtracting the minimum $\mathrm{P}$-wave duration (PWDmin) from the maximum P-wave duration (PWDmax). ECG, electrocardiogram.
The various $\mathrm{P}$-wave indices were then assessed for their impact on clinical end points of new-onset AF or recurrent stroke and also for their relation to echocardiographic markers of atrial cardiopathy such as LAVi. Continuous variables were presented as mean \pm standard deviation and compared using $t$ test. Categorical variables were presented as percentages and analysed using $\chi^{2}$ test or Fisher's exact test as appropriate. A multivariable logistic regression model adjusting for age, sex, hypertension, and diabetes mellitus was employed and results presented as adjusted odds ratios with $95 \%$ confidence intervals. All statistical tests were performed using IBM SPSS version 25 (IBM Corp., Armonk, NY, USA) where $p$ value of $<0.05$ was considered statistically significant.

\section{Results}

A total of 189 consecutive patients with ESUS were initially identified but 8 were excluded due to technical difficulties obtaining adequate echocardiographic views; the remaining 181 patients were included, forming the study cohort. Patients were predominantly Chinese $(69.2 \%)$ and had a mean age of $63.0 \pm 12.3$ years. The per- centage of male patients was $70.7 \%$ and the most common pre-existing comorbidity was hypertension (74.6\%). The patients were followed up for a median of 2.1 years (interquartile range, 1.4-2.8 years). The baseline characteristics of ESUS patients stratified by AF are shown in Table 1.

Seventy patients consented to and underwent prolonged cardiac monitoring with an implantable loop recorder (Medtronic Reveal LINQ; Medtronic Inc, Minneapolis, MN, USA). During follow-up, new-onset AF was detected in $14(7.7 \%)$ patients and $19(10.5 \%)$ developed recurrent ischaemic stroke. The rates of AF detection and recurrent ischaemic stroke in this ESUS cohort were comparable to previous studies [12, 13]. All 14 patients with new AF were started on anticoagulation and none had recurrent ischaemic stroke events. Between the AF and non-AF groups, there were no significant differences in demographics, comorbidities, or laboratory findings. More patients in the AF than non-AF group had implantable cardiac monitoring (Table 1). Echocardiographic left ventricular parameters and function were 
Table 1. Characteristics of study patients $(n=181)$

\begin{tabular}{|c|c|c|c|c|c|}
\hline Variable & $\begin{array}{l}\text { Total } \\
(n=181)\end{array}$ & $\begin{array}{l}\mathrm{AF} \\
(n=14)\end{array}$ & $\begin{array}{l}\text { Non-AF } \\
(n=167)\end{array}$ & Mean diff/OR & $p$ value \\
\hline Age, years & $63.0( \pm 12.3)$ & $65.3( \pm 11.9)$ & $62.8( \pm 12.4)$ & $2.53(-4.23$ to 9.30$)$ & 0.46 \\
\hline Male, $n(\%)$ & $128(70.7)$ & $8(57.1)$ & 120 (71.9) & $1.92(0.63-5.82)$ & 0.36 \\
\hline \multicolumn{6}{|l|}{ Ethnicity, $n(\%)$} \\
\hline Chinese & $125(69.1)$ & $14(100)$ & $111(66.5)$ & - & 0.08 \\
\hline Malay & $35(19.3)$ & $0(0)$ & $35(21.0)$ & & \\
\hline Others & 7 (3.9) & $0(0)$ & $7(4.2)$ & & \\
\hline \multicolumn{6}{|l|}{ Comorbidities, $n(\%)$} \\
\hline Hypertension & $135(74.6)$ & $12(85.7)$ & $123(73.7)$ & $2.15(0.46-9.97)$ & 0.52 \\
\hline Diabetes mellitus & $65(35.9)$ & $5(35.7)$ & $60(35.9)$ & $0.99(0.32-3.09)$ & 0.99 \\
\hline Dyslipidemia & $101(55.8)$ & $10(71.4)$ & $91(54.5)$ & $2.09(0.63-6.92)$ & 0.22 \\
\hline Ischaemic heart disease & $32(17.7)$ & $1(7.1)$ & $31(18.6)$ & $0.33(0.04-2.68)$ & 0.47 \\
\hline eGFR (mL/min; $N:>60)$ & $82.4( \pm 23.4)$ & $73.4( \pm 22.9)$ & $83.2( \pm 23.4)$ & $-9.82(-23.15$ to 5.31$)$ & 0.15 \\
\hline Fasting glucose, $\mathrm{mmol} / \mathrm{L}$ & $6.35( \pm 2.14)$ & $5.63( \pm 0.95)$ & $6.41( \pm 2.20)$ & $-0.78(-2.25$ to 0.69$)$ & 0.30 \\
\hline $\mathrm{HbA} 1 \mathrm{c}, \%$ & $6.66( \pm 2.02)$ & $6.72( \pm 2.30)$ & $6.66( \pm 2.01)$ & $0.06(-1.20$ to 1.31$)$ & 0.93 \\
\hline $\mathrm{LDL}-\mathrm{c}, \mathrm{mmol} / \mathrm{L}$ & $3.01( \pm 1.15)$ & $2.79( \pm 0.84)$ & $3.03( \pm 1.17)$ & $-0.24(-0.87$ to 0.40$)$ & 0.46 \\
\hline Multiple vascular territory of infarct, $n(\%)$ & $34(18.8)$ & $3(21.4)$ & $31(18.7)$ & $1.19(0.31-4.51)$ & 0.73 \\
\hline Prolonged cardiac monitoring with ICM, $n(\%)$ & $69(38.1)$ & $11(78.6)$ & $58(34.7)$ & $6.89(1.84-25.69)$ & 0.001 \\
\hline \multicolumn{6}{|l|}{ Echocardiographic parameters } \\
\hline LVEF $30-50 \%, n(\%)$ & $25(13.8)$ & $1(7.1)$ & $24(14.5)$ & $0.46(0.06-3.64)$ & 0.70 \\
\hline LA volume, $\mathrm{mL}$ & $47.4( \pm 17.8)$ & $62.3( \pm 22.0)$ & $46.1( \pm 16.9)$ & $16.17(3.30-29.04)$ & 0.001 \\
\hline LA volume index, $\mathrm{mL} / \mathrm{m}^{2}$ & $27.7( \pm 10.3)$ & $36.6( \pm 12.2)$ & $27.0( \pm 9.8)$ & $9.65(4.17-15.12)$ & 0.001 \\
\hline LA diameter, $\mathrm{mm}$ & $37.9( \pm 6.5)$ & $40.1( \pm 5.7)$ & $37.7( \pm 6.5)$ & $2.48(-1.07$ to 6.04$)$ & 0.17 \\
\hline LA diameter index, $\mathrm{mm} / \mathrm{m}^{2}$ & $22.0( \pm 4.3)$ & $23.9( \pm 4.0)$ & $21.8( \pm 4.3)$ & $2.09(-0.28$ to 4.46$)$ & 0.08 \\
\hline
\end{tabular}

OR, odds ratio; AF, atrial fibrillation; eGFR, estimated glomerular filtration rate; HbA1c, glycated haemoglobin; ICM, implantable cardiac monitor; LA, left atrial; LDL-C, low-density lipoprotein cholesterol; LVEDVi, left ventricular end-diastolic volume index; LVEF, left ventricular ejection fraction; LVMi, left ventricular mass index; TIA, transient ischaemic attack.

similar across the groups. The AF group had a significantly higher rate of atrial enlargement picked up from echocardiography as evidenced by parameters such as a higher LAVi.

The univariate and multivariate analyses are shown in Tables 2 and 3 respectively. Univariate analysis showed that PTFV1 was a significant predictor for the development of $\mathrm{AF}$ and this persisted in multivariate analyses (adjusted odds ratio 1.05, 95\% confidence interval 1.01-1.09, $p=0.02$ ). When ROC analysis was applied, the detection of AF was best predicted by PTFV1 $\geq 32.5 \mathrm{~ms}$, with sensitivity of $61.1 \%$ and specificity of $56.4 \%$ (Fig. 2). The other P-wave parameters showed varying effectiveness in the prediction for stroke or AF.
Abnormal P-wave axes, the presence of high grade IABs and PWDis were not found to be predictors for eventual diagnosis of AF on both univariate and multivariate analyses. PWD on the other hand was found to have a relation to recurrent stroke but not $\mathrm{AF}$ on univariate analysis when assessed as a categorical variable with a cut-off above $110 \mathrm{~ms}$, but the same relation was not observed after adjustment.

During the duration of follow-up, 19 patients (10.5\%) also developed a recurrent ischaemic stroke. Amongst these patients, none of the P-wave indices evaluated were predictive of subsequent stroke. 


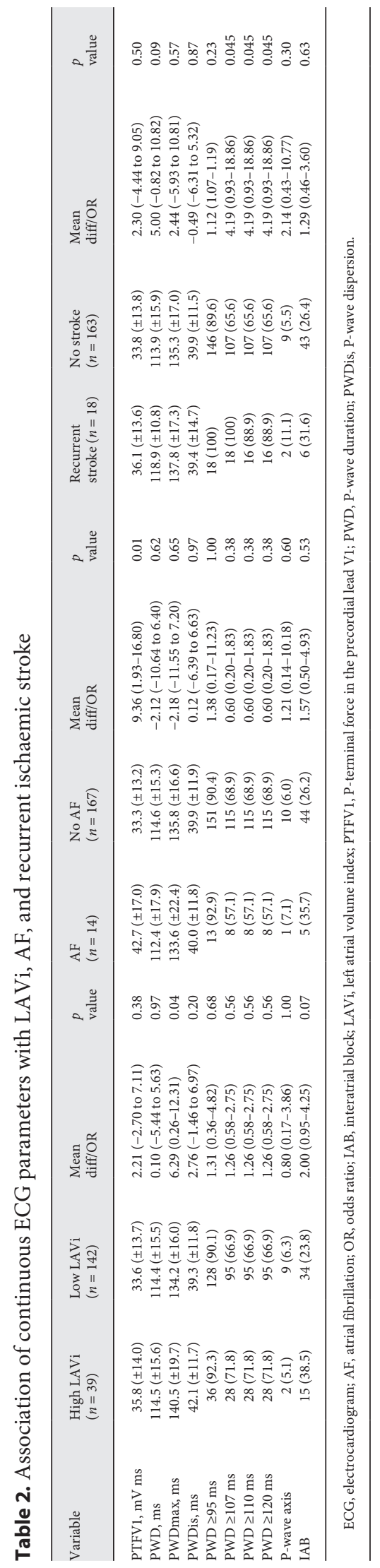

Table 3. Multivariable logistic regression analysis of ECG predictors of outcomes

\begin{tabular}{lll}
\hline Variable & OR $(95 \% \mathrm{CI})$ & $p$ value \\
\hline Prediction of AF & & \\
PTFV1 & $1.05(1.01-1.09)$ & 0.02 \\
PWD & $0.99(0.95-1.03)$ & 0.55 \\
PWDmax & $0.99(0.96-1.03)$ & 0.57 \\
\hline Prediction of recurrent stroke & & \\
PTFV1 & $1.01(0.98-1.05)$ & 0.56 \\
PWD & $1.02(0.99-1.05)$ & 0.28 \\
PWDmax & $1.01(0.98-1.04)$ & 0.57 \\
\hline
\end{tabular}

Adjusted for age, sex, hypertension, and diabetes mellitus. ECG, electrocardiogram; OR, odds ratio; AF, atrial fibrillation; IAB, interatrial block; LAVi, left atrial volume index; PTFV1, P-terminal force in the precordial lead V1; PWD, P-wave duration; PWDis, P-wave dispersion.

\section{Discussion}

In this study, $\mathrm{P}$-wave indices were analysed for their utility in predicting AF and recurrent stroke in a cohort of ESUS patients. PTFV1 was associated with occult AF detection but not with recurrent ischaemic strokes.

A hypothesis surrounding ESUS is that undiagnosed cardioembolism contributes to a large proportion of cryptogenic strokes and such strokes could benefit from anticoagulation [2]. Unfortunately, 2 recent large randomized controlled trials did not observe a benefit for direct oral anticoagulation towards preventing recurrent stroke in patients with ESUS $[12,13]$. However, subgroup analyses found that stroke risk can be mitigated by direct oral anticoagulation in a subset of patients with an enlarged left atrium [14]. This lends to the belief that there might be a subgroup of patients within ESUS whose occult embolic mechanisms are likely to respond to anticoagulant therapy, specifically patients with paroxysmal AF which has not been picked up yet or atrial cardiopathy without AF. Identifying these patients who might respond to oral anticoagulation is therefore key. While echocardiographic evaluation remains a common part of standard evaluation for stroke patients, it remains relatively costly and may not always be readily available in clinical practice. The ECG, however, is inexpensive and readily available. P-wave analysis could therefore be a more easily accessible means of gathering information about the predisposition of patients to AF and thereby guide the provision of tailored preventive therapy. 


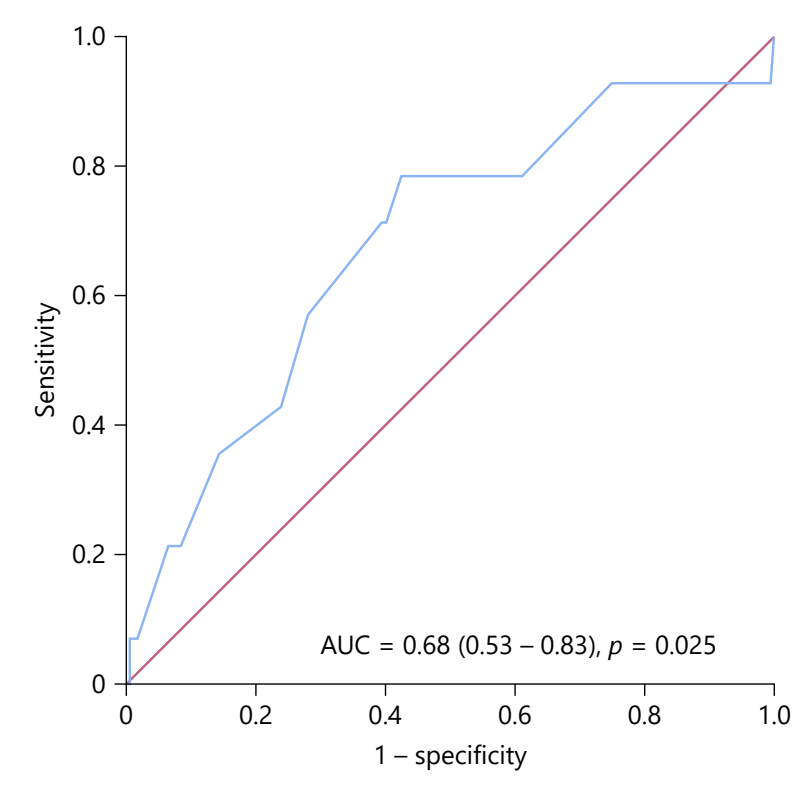

Fig. 2. Receiver operator curve for PTFV1 as a predictor for AF. The AUC was $0.68(0.53-0.83), p=0.025$, optimized cut-off was $32.5 \mathrm{mV}$ ms, with sensitivity of $61.1 \%$, specificity of $56.4 \%$, PPV of $10.3 \%$, and NPV of $94.7 \%$. AF, atrial fibrillation; AUC, area under the curve; NPV, negative predictive value; $\mathrm{PPV}$, positive predictive value; PTFV1, P-terminal force in the precordial lead V1.

Previous studies have identified several $\mathrm{P}$-wave indices that are markers of LA dysfunction and associated with ischaemic stroke with or without AF [17-21, 23-25]. However, these markers of atrial cardiopathy remain most strongly associated with embolic strokes and have not been validated thoroughly in an ESUS cohort. Our study with an ESUS cohort finds PTFV1 to correlate with subsequent $\mathrm{AF}$ detection but the presence of IAB, abnormal $\mathrm{P}$-wave axis, and prolonged PWD and PWDis were not found to be significant. Interestingly while PTFV1 was found to correlate with AF detection, it was not associated with recurrent strokes. This could possibly be because our results were partially attenuated by the fact that all the patients with detected AF were started on anticoagulation and therefore avoided developing a subsequent stroke. This could support the hypothesis that ESUS is a heterogenous entity and there might be a significant group of patients who might have occult embolic mechanisms not related to AF or underlying atrial cardiopathy. These would include conditions such as non-stenosing large-artery atherosclerosis and non-atherosclerotic vasculopathies among others [26]. Anticoagulation could prove superior to antiplatelet therapy for cases with first group of em-

P-Wave Markers and Atrial Fibrillation in Embolic Stroke of Undetermined Source bolic causative mechanisms but not those with the latter atherosclerotic causes, suggesting the need for better risk stratification and personalized therapy.

We do note that overall, the correlation between these $\mathrm{P}$-wave indices with eventual AF diagnosis and stroke in this ESUS population was weaker as compared to that reported in previous studies that investigated undifferentiated cohorts of patients with ischaemic stroke. This could point to the natural history of AF development where there is atrial enlargement, atrial fibrosis, endothelial dysfunction, and ultimately AF [11]. Those cases with more overt atrial cardiopathy would likely have manifested AF earlier and have been detected on the initial work-up and therefore classified as a cardioembolic stroke instead of ESUS to begin with.

While recognizing the potential of $\mathrm{P}$-wave indices in predicting atrial cardiopathy, we do have to be cognizant of the challenges in identifying and interpreting these markers. The P-wave is a low voltage signal on the ECG and its accurate interpretation is challenging especially in cases where there is significant baseline noise. This could lead to challenges identifying the true start and end of the $\mathrm{P}$-wave as well as its amplitude. Given that some of these $\mathrm{P}$-wave indices require further mathematical functions such as multiplication that is required in PTFV1, this can result in the potential magnification of errors [27]. These aforementioned factors could have contributed to some of the heterogeneity in the evidence surrounding the associations of $\mathrm{P}$-wave indices and AF detection as well as stroke in literature $[21,23]$.

To mitigate this challenge, our study aimed to be as realistic as possible to evaluate these ECG parameters on their potential application in clinical practice. Firstly, the parameters chosen are easily identifiable and measured directly from the ECG strip. We have, therefore, avoided studying more complex parameters that would require specialized software such as P-wave area assessment [28]. We also chose to assess the ECGs on direct visual inspection, albeit under magnification, without the use of more specialized tools such as electronic calipers to ensure that the chosen parameters could be assessed easily and readily in daily practice. As the results show, even with these relative limitations, we were able to identify these $\mathrm{P}$-wave parameters with sufficient accuracy to be potentially useful towards risk stratification in ESUS patients. Going forward, computerized algorithms that would be able to automatically assess for these parameters might be able to improve the accuracy of assessing such $\mathrm{P}$-wave parameters, improving their accuracy and applicability for daily use. 


\section{Limitations}

We acknowledge several limitations in this study. Although our study has a moderate sample size for an ESUS cohort, its retrospective nature means that we could only show association but not causation. This was a singlecentre study which may limit generalizability. In addition, less than half of the patients were monitored with prolonged cardiac monitoring using an implantable loop recorder, which may have resulted in under-detection of occult AF. This was because implantation of a loop recorder in our hospital required co-payment, and most patients declined due to financial issues.

\section{Conclusion}

PTFV1 is associated with AF detection but not recurrent strokes in this cohort of ESUS patients. P-wave indices such as PTFV1 might be practical markers in identifying patients who potentially benefit from anticoagulation, but these observations need to be studied in larger prospective studies.

\section{Statement of Ethics}

This retrospective study involving human participants was in accordance with the ethical standards of the institutional and national research committee and with the 1964 Helsinki Declaration and its later amendments or comparable ethical standards. The
Domain-Specific Review Board, National Healthcare Group, Singapore, approved this study. Requirements for written informed consent from subjects were waived due to the retrospective nature of this study.

\section{Conflict of Interest Statement}

The authors declare that they have no conflicts of interest.

\section{Funding Sources}

Ching-Hui Sia was supported by the National University of Singapore Yong Loo Lin School of Medicine's Junior Academic Faculty Scheme.

\section{Author Contributions}

Tony Y.W. Li, Benjamin Y.Q. Tan, Ching-Hui Sia, Swee Chong Seow, Pipin Kojodjojo, Vijay K. Sharma, and Leonard L.L. Yeo contributed to conception or design of the work. Benjamin Y.Q. Tan, Tony Y.W. Li, Jamie S.Y. Ho, Ching-Hui Sia, Aloysius S. Leow, Vijay K. Sharma, and Leonard L.L. Yeo contributed to data acquisition, data analysis, and/or interpretation. Benjamin Y.Q. Tan, Tony Y.W. Li, Ching-Hui Sia, Mayank Dalakoti, Mark Y. Chan, Swee Chong Seow, and Pipin Kojodjojo contributed to drafting the article. Tony Y.W. Li, Benjamin Y.Q. Tan, Jamie S.Y. Ho, Ching-Hui Sia, Aloysius S. Leow, Mayank Dalakoti, Mark Y. Chan, Bernard P.L. Chan, Hock Luen Teoh, Swee Chong Seow, Pipin Kojodjojo, Vijay K. Sharma, and Leonard L.L. Yeo contributed to critical revision of the article. All authors had final approval of the version to be published. Benjamin Y.Q. Tan, Ching-Hui Sia, and Leonard L.L. Yeo were responsible for overall supervision.

\section{References}

1 Krishnamurthi RV, Feigin VL, Forouzanfar MH, Mensah GA, Connor M, Bennett DA, et al. Global and regional burden of first-ever ischaemic and haemorrhagic stroke during 1990-2010: findings from the Global Burden of Disease Study 2010. Lancet Glob Health. 2013 Nov; 1(5):e259-81.

2 Hart RG, Catanese L, Perera KS, Ntaios G, Connolly SJ. Embolic stroke of undetermined source: a systematic review and clinical update. Stroke. 2017 Apr;48(4):867-72.

3 Marnane M, Duggan CA, Sheehan OC, Merwick A, Hannon N, Curtin D, et al. Stroke subtype classification to mechanism-specific and undetermined categories by TOAST, A$\mathrm{S}-\mathrm{C}-\mathrm{O}$, and causative classification system: direct comparison in the North Dublin population stroke study. Stroke. 2010 Aug;41(8): 1579-86.

4 Hart RG, Diener H-C, Coutts SB, Donald Easton J, Granger CB, O’Donnell MJ, et al. Embolic strokes of undetermined source: the case for a new clinical construct. Lancet Neurol. 2014 Apr;13(4):429-38.

5 Sposato LA, Cipriano LE, Saposnik G, Ruíz Vargas E, Riccio PM, Hachinski V. Diagnosis of atrial fibrillation after stroke and transient ischaemic attack: a systematic review and meta-analysis. Lancet Neurol. 2015 Apr;14(4): 377-87.

6 Seet RC, Friedman PA, Rabinstein AA. Prolonged rhythm monitoring for the detection of occult paroxysmal atrial fibrillation in ischemic stroke of unknown cause. Circulation. 2011 Jul 26;124(4):477-86.

7 Sinha AM, Diener H-C, Morillo CA, Sanna T, Bernstein RA, Lazzaro VD, et al. Cryptogenic stroke and underlying atrial fibrillation (CRYSTAL AF): design and rationale. Am Heart J. 2010 Jul;160(1):36-41.e1.

8 Yushan B, Tan BYQ, Ngiam NJ, Chan BPL, Luen TH, Sharma VK, et al. Association between bilateral infarcts pattern and detection of occult atrial fibrillation in embolic stroke of undetermined source (ESUS) patients with insertable cardiac monitor (ICM). J Stroke Cerebrovasc Dis. 2019 Sep; 28(9):2448-52.

9 Liantinioti C, Palaiodimou L, Tympas K, Parissis J, Theodorou A, Ikonomidis I, et al. Potential utility of neurosonology in paroxysmal atrial fibrillation detection in patients with cryptogenic stroke. J Clin Med. 2019;8(11): 2002.

10 Tsivgoulis G, Katsanos AH, Köhrmann M, Caso V, Perren F, Palaiodimou L, et al. Duration of implantable cardiac monitoring and detection of atrial fibrillation in ischemic stroke patients: a systematic review and meta-analysis. J Stroke. 2019 Sep;21(3): 302-11.

11 Heijman J, Voigt N, Nattel S, Dobrev D. Cellular and molecular electrophysiology of atrial fibrillation initiation, maintenance, and progression. Circ Res. 2014 Apr 25;114(9): 1483-99. 
12 Hart RG, Sharma M, Mundl H, Kasner SE, Bangdiwala SI, Berkowitz SD, et al. Rivaroxaban for stroke prevention after embolic stroke of undetermined source. N Engl J Med. 2018 Jun 7;378(23):2191-201.

13 Diener HC, Sacco RL, Easton JD, Granger CB, Bernstein RA, Uchiyama S, et al. Dabigatran for prevention of stroke after embolic stroke of undetermined source. N Engl J Med. 2019 May 16;380(20):1906-17.

14 Healey JS, Gladstone DJ, Swaminathan B, Eckstein J, Mundl H, Epstein AE, et al. Recurrent stroke with rivaroxaban compared with aspirin according to predictors of atrial fibrillation: secondary analysis of the NAVIGATE ESUS randomized clinical trial. JAMA Neurol. 2019 Jul;76(7):764-73.

15 Ho J, Tan B, Sia C-H. Letter by Ho et al regarding article, left atrial volume index is associated with cardioembolic stroke and atrial fibrillation detection after embolic stroke of undetermined source. Stroke. 2019 Sep 20; 50(11):e331.

16 Tan BYQ, Ho JSY, Sia C-H, Boi Y, Foo ASM, Dalakoti $\mathrm{M}$, et al. Left atrial volume index predicts new-onset atrial fibrillation and stroke recurrence in patients with embolic stroke of undetermined source. Cerebrovasc Dis. 2020; 49(3):285-91.
17 Okin PM, Kamel H, Kjeldsen SE, Devereux RB. Electrocardiographic left atrial abnormalities and risk of incident stroke in hypertensive patients with electrocardiographic left ventricular hypertrophy. J Hypertens. 2016 Sep;34(9):1831-7.

18 Dogan U, Dogan EA, Tekinalp M, Tokgoz OS, Aribas A, Akilli $\mathrm{H}$, et al. P-wave dispersion for predicting paroxysmal atrial fibrillation in acute ischemic stroke. Int J Med Sci. 2012;9(1):108-14.

19 Lorbar M, Levrault R, Phadke JG, Spodick $\mathrm{DH}$. Interatrial block as a predictor of embolic stroke. Am J Cardiol. 2005 Mar 1;95(5): 667-8.

20 Maheshwari A, Norby FL, Soliman EZ, Koene RJ, Rooney MR, O'Neal WT, et al. Abnormal $\mathrm{P}$-wave axis and ischemic stroke: the ARIC study (Atherosclerosis risk in communities). Stroke. 2017;48(8):2060-5.

21 He J, Tse G, Korantzopoulos P, Letsas KP, AliHasan-Al-Saegh S, Kamel H, et al. P-wave indices and risk of ischemic stroke: a systematic review and meta-analysis. Stroke. 2017 Aug; 48(8):2066-72.

22 Lang RM, Badano LP, Mor-Avi V,, Afilalo J, Armstrong A, Ernande L, L, et al. Recommendations for cardiac chamber quantification by echocardiography in adults: an update from the American society of echocardiography and the European association of cardiovascular imaging. Eur Heart J Cardiovasc Imaging. 2015;16(3):233-71.
23 Huang Z, Zheng Z, Wu B, Tang L, Xie X, Dong $\mathrm{R}$, Luo $\mathrm{Y}$, et al. Predictive value of $\mathrm{P}$ wave terminal force in lead V1 for atrial fibrillation: a meta-analysis. Ann Noninvasive Electrocardiol. 2020 Feb 5:25(4):e12739.

24 Mendieta G, Guasch E, Weir D, Aristizabal D, Escobar-Robledo LA, Llull L, et al. Advanced interatrial block: a predictor of covert atrial fibrillation in embolic stroke of undetermined source. J Electrocardiol. 2020 Jan-Feb; 58:113-8.

25 Vural MG, Cetin S, Yilmaz M, Akdemir R, Gunduz H. Relation between left atrial remodeling in young patients with cryptogenic stroke and normal inter-atrial anatomy. J Stroke. 2015 Sep;17(3):312-9.

26 Kamel H, Merkler AE, Iadecola C, Gupta A, Navi BB. Tailoring the approach to embolic stroke of undetermined source: a review. JAMA Neurol. 2019 Jul 1;76(7):855-61.

27 Sajeev JK, Koshy AN, Dewey H, Kalman JM, Bhatia M, Roberts L, et al. Poor reliability of $\mathrm{P}$-wave terminal force $\mathrm{V} 1$ in ischemic stroke. J Electrocardiol. 2019 Jan-Feb;52:47-52.

28 Weinsaft JW, Kochav JD, Kim J, Gurevich S, Volo SC, Afroz A, et al. P wave area for quantitative electrocardiographic assessment of left atrial remodeling. PLoS One. 2014;9(6): e99178. 